\title{
BMJ Open Establishing the Aus-ROC Australian and New Zealand out-of-hospital cardiac arrest Epistry
}

Ben Beck, ${ }^{1}$ Janet Bray, ${ }^{1,2}$ Karen Smith, ${ }^{1,3,4}$ Tony Walker, ${ }^{3}$ Hugh Grantham, ${ }^{5,6}$ Cindy Hein, ${ }^{5,6}$ Melanie Thorrowgood, ${ }^{6}$ Anthony Smith, ${ }^{7}$ Tony Smith, ${ }^{8}$ Bridget Dicker, ${ }^{8,9}$ Andy Swain, ${ }^{10}$ Mark Bailey, ${ }^{10}$ Emma Bosley, ${ }^{11,12}$ Katherine Pemberton, ${ }^{11}$ Peter Cameron, ${ }^{1,13}$ Graham Nichol, ${ }^{14}$ Judith Finn, ${ }^{1,2,4,7}$ on behalf of the Aus-ROC Steering Committee

To cite: Beck B, Bray J, Smith $\mathrm{K}$, et al. Establishing the Aus-ROC Australian and New Zealand out-of-hospital cardiac arrest Epistry. BMJ Open 2016;6:e011027. doi:10.1136/bmjopen-2016011027

- Prepublication history and additional material is available. To view please visit the journal (http://dx.doi.org/ 10.1136/bmjopen-2016011027).

Received 1 January 2016 Accepted 12 February 2016

CrossMark

For numbered affiliations see end of article.

Correspondence to Professor Judith Finn; judith.finn@curtin.edu.au

\section{ABSTRACT}

Introduction: Out-of-hospital cardiac arrest (OHCA) is a global health problem with low survival. Regional variation in survival has heightened interest in combining cardiac arrest registries to understand and improve OHCA outcomes. While individual OHCA registries exist in Australian and New Zealand ambulance services, until recently these registries have not been combined. The aim of this protocol paper is to describe the rationale and methods of the Australian Resuscitation Outcomes Consortium (Aus-ROC) OHCA epidemiological registry (Epistry).

Methods and analysis: The Aus-ROC Epistry is designed as a population-based cohort study. Data collection started in 2014. Six ambulance services in Australia (Ambulance Victoria, SA Ambulance Service, St John Ambulance Western Australia and Queensland Ambulance Service) and New Zealand (St John New Zealand and Wellington Free Ambulance) currently contribute data. All OHCA attended by ambulance, regardless of aetiology or patient age, are included in the Epistry. The catchment population is approximately 19.3 million persons, representing $63 \%$ of the Australian population and $100 \%$ of the New Zealand population. Data are collected using Utstein-style definitions. Information incorporated into the Epistry includes demographics, arrest features, ambulance response times, treatment and patient outcomes. The primary outcome is 'survival to hospital discharge', with 'return of spontaneous circulation' as a key secondary outcome.

Ethics and dissemination: Ethics approval was independently sought by each of the contributing registries. Overarching ethics for the Epistry was provided by Monash University HREC (Approval No. CF12/39382012001888). A population-based OHCA registry capturing the majority of Australia and New Zealand will allow risk-adjusted outcomes to be determined, to enable benchmarking across ambulance providers, facilitate the identification of system-wide strategies associated with survival from OHCA, and allow monitoring of temporal trends in process and outcomes to improve patient care. Findings will be shared with participating ambulance services and the academic community.

\section{Strengths and limitations of this study}

- The Australian Resuscitation Outcomes Consortium (Aus-ROC) epidemiological registry (Epistry) is a population-based out-of-hospital cardiac arrest (OHCA) registry with a capture region encompassing the majority of Australia and all of New Zealand.

- The Epistry provides an ability to define consistent primary and secondary outcome measures to compare the effects of regional and ambulance service variations and understand factors associated with improved survival from OHCA.

- Combining data from multiple regions has inherent challenges, including variation in the variables collected by each of the participating ambulance services.

\section{INTRODUCTION}

Out-of-hospital cardiac arrest (OHCA) is a global health problem. ${ }^{1}$ Despite over 50 years since the advent of cardiopulmonary resuscitation, ${ }^{2}$ survival remains low with reported rates between $0.6 \%$ and $25 \% .{ }^{3}$ Recognition of the variation in OHCA survival rates between regions has heightened interest in the development of multicentre cardiac arrest registries to understand and improve OHCA outcomes.

Individual OHCA registries have been established by ambulance services in most Australian states and across the whole of New Zealand. However, until recently, combining these registries has been hindered by a lack of infrastructure to host the data and to ensure a consensus of definitions and standardisation. As illustrated by the success of international groups, such as the Resuscitation Outcomes Consortium (ROC), ${ }^{4}$ the combining of registries across a larger region facilitates: the understanding of regional differences in the OHCA incidence and outcomes; the 


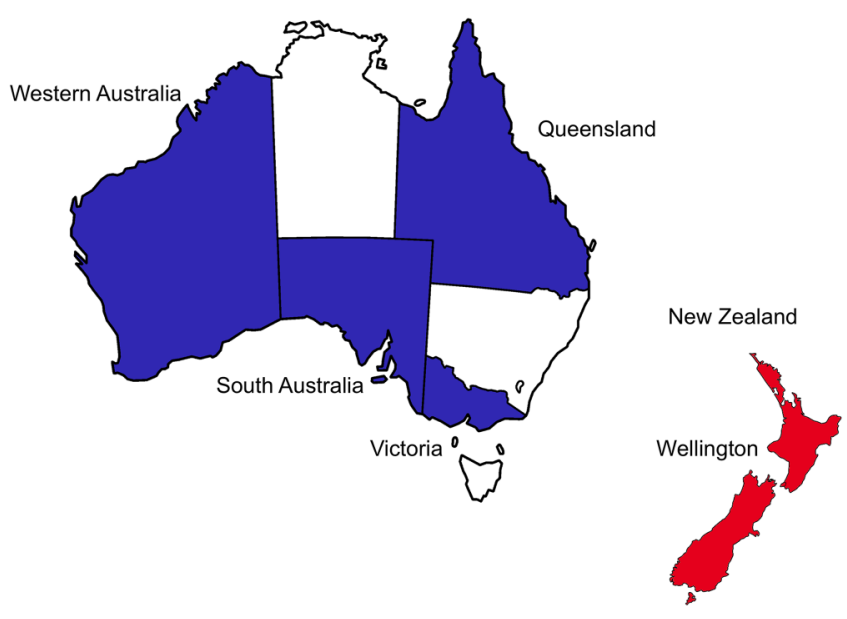

Figure 1 Map of Aus-ROC Epistry highlighting the captured regions in Australia and New Zealand (shown in colour).

influence of different ambulance service structures; and the patient, ambulance service and period factors associated with survival. ${ }^{5-7}$

\section{AIMS AND OBJECTIVES OF THE PROJECT}

The Australian ROC (Aus-ROC) ${ }^{8}$ was established as a National Health and Medical Research Council (NHRMC) Centre of Research Excellence in 2011 to increase research capacity aimed at improving OHCA survival and outcomes. One of the major objectives of this consortium was to develop an Australian and New Zealand cardiac arrest epidemiological registry (Epistry) to enhance and inform coordinated OHCA outcomes, research and evaluation. This paper describes the rationale and methods of the Aus-ROC Epistry.

\section{METHODS}

Study design

The Aus-ROC Epistry is designed as a population-based cohort study. Data collection started in 2014. Six established cardiac arrest registries, four in Australia and two in New Zealand, contribute data to the Epistry (figure 1). All six cardiac arrest registries are maintained by ambulance services: Ambulance Victoria, SA Ambulance Service, St John Ambulance Western Australia, Queensland Ambulance Service, St John New Zealand and Wellington Free Ambulance. Approximately 19.3 million persons are served by these ambulance services (table 1); thus, the Epistry represents approximately $63 \%$ of the Australian population (total population of 23.5 million $^{9}$ ) and $100 \%$ of the New Zealand population (total population of 4.5 million).$^{10}$

Demographic and socioeconomic characteristics of each of the regions in Australia and New Zealand are shown in online supplementary appendices 2 and 3, respectively. ${ }^{17-19}$ Australian regions participating in the Epistry demonstrated similar characteristics to those of the broader Australian population. While some regions not currently participating in the Epistry demonstrated variations in the proportion of the population who were Aboriginal and Torres Strait Island peoples and those living in 'major cities', these regions (Australian Capital Territory, Northern Territory and Tasmania) had small total populations that reflected a combined $4.9 \%$ of the Australian population.

Differences in the classification of geographical regions did not facilitate comparisons between Australia and New Zealand. Alternate metrics may be required when making comparisons of rurality between the two countries.

\section{Inclusion criteria}

All OHCA attended by ambulance, regardless of aetiology, are included in the Epistry. All deaths attended by ambulance are classified as OHCA and thus included in the Epistry.

\section{Data capture}

Capture of OHCA cases varies between ambulance services. A combination of electronic queries of patient care records (PCRs), manual sorting through PCRs, staff standardised reporting and dispatch codes are used to identify OHCA cases. For those ambulance services that use electronic queries, subsequent manual searches are conducted by all services to maximise case capture. Hospital

Table 1 Description of the six participating Australian Resuscitation Outcomes Consortium (Aus-ROC) regions

\begin{tabular}{llrr}
\hline Region & Ambulance service & $\begin{array}{c}\text { Service area } \\
\text { population }\end{array}$ & $\begin{array}{c}\text { Geographic } \\
\text { area (ha) }\end{array}$ \\
\hline South Australia, Australia & SA Ambulance Service & $1685714^{9}$ & $98417966^{11}$ \\
Victoria, Australia & Ambulance Victoria & $5841667^{9}$ & $22749552^{12}$ \\
Western Australia, Australia & St John Ambulance Western Australia & $2573389^{9}$ & $252641786^{13}$ \\
Queensland, Australia & Queensland Ambulance Service & $4722447^{9}$ & $172582593^{14}$ \\
$\quad$ Australian Registries & & 14823217 & 121809304 \\
New Zealand (excluding Wellington) & St John New Zealand & $4018370^{10}$ & $26152195^{15}$ \\
Wellington, New Zealand & Wellington Free Ambulance & $491380^{10}$ & $813005^{16}$ \\
$\quad$ New Zealand Registries & & 4509750 & 26965200 \\
Total & & $\mathbf{1 9 3 3 2 9 6 7}$ & $\mathbf{5 7 3} \mathbf{3 5 7 0 9 7}$
\end{tabular}

Australia population=23 490736 , Australia geographic area $=758848540.5$ ha, ${ }^{12}$ New Zealand population $=4509750,{ }^{10}$ New Zealand geographic area=26 965200 ha. $^{15}$ 
outcomes data are commonly obtained through linkage with hospital records and state-based death registries. In one region, hospital outcomes are obtained through linkage with the state Department of Health data.

\section{Variables}

Data across all participating sites are collected in accordance with Utstein-style definitions, ${ }^{20}$ although some variation in the coding of these items exists. Recoding of each variable is conducted to ensure uniformity in the variables collected. Data include information on the OHCA event, demographics, arrest features, response times, treatment and outcome. Variables were identified by consensus among representatives from participating ambulance services (table 2). All variables are collected by each of the six participating ambulance services.

The primary outcome measure is 'survival to hospital discharge', with 'return of spontaneous circulation' as a key secondary outcome. These measures are considered 'core' outcomes according to the Utstein template. ${ }^{20}$ While neurological outcome and long-term quality of life measurements are important patient-centred outcomes, they are not presently collected across all sites, and thus these measures are not currently included in the Epistry.

\section{Ethical considerations}

Ethics approval was independently sought by each of the contributing registries. Overarching ethics for the Epistry was provided by Monash University HREC (Approval No. CF12/3938-2012001888). Data from each of the contributing registries will be provided in reidentifiable form with a unique identifier should the need arise to cross-check individual cases.

\section{Data management and governance}

The Epistry is coordinated and located at the Aus-ROC administrative base in the School of Public Health and Preventive Medicine at Monash University, Australia. Each participating ambulance service is responsible for regulating case ascertainment, data capture and quality control. Data upload is web-based and is stored on a secure server at Monash University. Data are then recoded by Epistry staff at Monash University for consistency with the Epistry data dictionary and then combined to form the Epistry.

An Epistry Management Committee (EMC) was established to develop processes and protocols, and oversee all ongoing aspects of the Epistry governance. The committee comprises representatives from each of the contributing ambulance services and Aus-ROC investigators. The Epistry consensus governance document guides the committee on the agreed use of the Epistry data; while maintaining data security and ethical principles.

\section{Statistical analyses}

Based on previously reported incidence of OHCA in our region, ${ }^{21}$ we estimate that the Epistry will capture approximately 14000 patients with OHCA annually. We anticipate

\begin{tabular}{|c|c|}
\hline Group & Variable \\
\hline Case overview & $\begin{array}{l}\text { Unique Aus-ROC case identifier } \\
\text { Event date } \\
\text { Longitude coordinate of event } \\
\text { Latitude coordinate of event }\end{array}$ \\
\hline Demographics & $\begin{array}{l}\text { Age } \\
\text { Age qualifier } \\
\text { Sex } \\
\text { Location of arrest }\end{array}$ \\
\hline Arrest features & $\begin{array}{l}\text { Witnessed arrest } \\
\text { Bystander CPR } \\
\text { First arresting rhythm } \\
\text { Aetiology of arrest }\end{array}$ \\
\hline $\begin{array}{l}\text { Dates and } \\
\text { times }\end{array}$ & $\begin{array}{l}\text { Date and time call received } \\
\text { Date and time arrive at scene (first) } \\
\text { Date and time depart scene (transporting } \\
\text { ambulance) } \\
\text { Date and time arrive hospital } \\
\text { (transporting ambulance) }\end{array}$ \\
\hline Treatment & $\begin{array}{l}\text { Ambulance service resuscitation attempt } \\
\text { Ambulance service defibrillation } \\
\text { Defibrillation before ambulance service } \\
\text { Total number of shocks } \\
\text { Airway (final) } \\
\text { Mechanical CPR }\end{array}$ \\
\hline Outcomes & $\begin{array}{l}\text { Any ROSC } \\
\text { Scene outcome } \\
\text { Survived event (ROSC on hospital } \\
\text { handover) } \\
\text { Died } \\
\text { Discharged alive } \\
\text { Date of discharge/death } \\
\text { Discharge disposition } \\
\text { Receiving hospital (PCl capability) }\end{array}$ \\
\hline
\end{tabular}

using descriptive statistics to summarise baseline characteristics and survival outcomes. Annual crude and age-sex-adjusted incidence rates will be calculated using population figures from the Australian Bureau of Statistics and Statistics New Zealand. Logistic regression will be used to investigate factors associated with key outcome measures with ambulance services as random effects.

\section{Reporting}

Publically available reports will be generated on an annual basis, in which ambulance services will be de-identified. As a component of quality improvement, benchmarking reports will be confidentially provided to each of the participating ambulance services comparing their risk-adjusted outcomes with the combined outcomes of the other ambulance services participating in the Aus-ROC Epistry. Findings will also be reported in peer-reviewed publications. 


\section{DISCUSSION}

The Aus-ROC Epistry was established with the aim of understanding regional, ambulance service and treatment factors associated with improved OHCA survival and outcomes. The scope of the Epistry will enable a range of important research questions to be answered, including understanding regional variations in outcome, the impact of variations in the provision of treatment for OHCA between ambulance services, temporal changes in incidence and outcomes, and the impact of changes in clinical guidelines and clinical trials, as well as increased clinical trial efficiency. The Epistry will allow risk-adjusted outcomes to be determined, to enable benchmarking across providers and identification of system-wide strategies associated with survival for patients with OHCA in Australia and New Zealand.

Variations have been identified in the incidence and outcomes of OHCA across geographical regions in Australia $^{21} 22$ and internationally. ${ }^{2-25}$ To improve outcomes for these patients, there is a need to identify the underlying causes of these variations, which may include differences in the underlying risk of OHCA, and ambulance service structure, function and resuscitation practices; or simply case definitions. For example, other multicentre cardiac arrest registries have investigated regional variation in: the incidence and outcomes of OHCA, ${ }^{23} 2627$ temporal trends in survival, ${ }^{6}$ rates of bystander cardiopulmonary resuscitation $(\mathrm{CPR}),{ }^{28}$ treatment and transport practices, ${ }^{7}$ and the association between receiving hospital characteristics and survival. ${ }^{29}$ The Epistry will serve as a framework to address similar questions and identify factors associated with regional variation in survival. The longitudinal nature of the Epistry enables pre-post studies to assess the effect of changes in practice, such as examining whether survival from OHCA improves following the implementation of guideline updates. ${ }^{30}$ The Epistry will also facilitate the evaluation of clinical trials by providing infrastructure to capture and combine prehospital and in-hospital data across multiple regions in a standardised manner. It is intended to eventually broaden the telephone assessment of quality-of-life (using validated instruments) of OHCA survivors that is conducted by the Victorian Ambulance Cardiac Arrest Registry ${ }^{31}$ and in Aus-ROC clinical trials, ${ }^{32}$ and use this information to study patient-centred outcomes, as well as economic evaluation of resuscitation interventions.

The Aus-ROC Epistry has been modelled on the successful ROC Epistry. ${ }^{4}$ In comparison to ROC who rely on data from 264 emergency medical service agencies, ${ }^{4}$ one of the strengths of Aus-ROC is the contribution of data from only six ambulance services. Individually, these ambulance services cover large geographical areas and populations, and represent both metropolitan and rural regions, facilitating understanding of variability in the ambulance service systems and the impact that such variability has on patient outcomes. When compared with other multicentre registries that capture smaller proportions of their relevant population, the capture population of the Epistry represents the majority of Australia and New Zealand and is therefore more likely to be representative of the broader population. With significant heterogeneity in the reporting of OHCA outcomes, ${ }^{33}$ another strength of the Aus-ROC Epistry is the ability to define consistent primary and secondary outcome measures to compare the effect of regional and ambulance service variations. Similarly, having a consistent and uniform denominator when defining incidence and survival in OHCA enables valid comparisons across regions.

The sensitive nature of OHCA data, particularly for ambulance services, presents challenges when establishing multicentre cardiac arrest registries. ${ }^{34}$ These challenges include data ownership, access security and use. In establishing the Epistry, memorandums of understanding were developed between the lead academic institution and each of the participating ambulance services. These agreements, combined with the Epistry governance document, provide clear guidelines on the ownership, use and dissemination of Epistry data.

Combining data from multiple regions has inherent challenges. Variation existed in the variables collected by each of the participating ambulance services, which reduced the number of core variables that were collected for the Epistry. However, it is hoped that over time, all participating sites will be able to collect additional variables, such as 'neurological outcome' and other resuscitation practices and interventions. A survey of the participating ambulance services is currently underway to evaluate variation in the treatment and provision of service between Epistry sites. This survey will also collect data on case ascertainment and quality control measures within each of the participating sites. Aus-ROC also has a vision to extend the current network of participating ambulance services to include all Australian ambulance services. While four Australian ambulance services not currently participating in the Epistry have been invited to contribute data (NSW Ambulance, St John Ambulance Northern Territory, ACT Ambulance, Ambulance Tasmania), these service-based OHCA registries are in various stages of development. It is hoped that these registries will be able to contribute data in the future, and thus the Epistry will capture the prevalence of OHCA in the entire Australian population-as is currently possible for New Zealand.

\section{SUMIMARY}

The Aus-ROC Epistry is a population-based OHCA registry with a capture region encompassing the majority of Australia and all of New Zealand. Combining OHCA data from six sites will facilitate an understanding of the factors that are associated with improved survival from OHCA and will allow monitoring of temporal trends in process and outcome. Furthermore, the Epistry provides infrastructure to enhance the coordination of clinical trials and improve collaboration between ambulance services, thus increasing capacity to improve outcomes of patients with OHCA in Australia and New Zealand. 
Author affiliations

${ }^{1}$ Department of Epidemiology and Preventive Medicine, Monash University, Melbourne, Victoria, Australia

2Prehospital, Resuscitation and Emergency Care Research Unit (PRECRU), Curtin University, Perth, Western Australia, Australia

${ }^{3}$ Ambulance Victoria, Doncaster, Victoria, Australia

${ }^{4}$ Discipline of Emergency Medicine, University of Western Australia, Perth, Western Australia, Australia

${ }^{5}$ Flinders University, Adelaide, South Australia, Australia

${ }^{6}$ SA Ambulance Service, Eastwood, South Australia, Australia

${ }^{7}$ St John Ambulance Western Australia, Perth, Western Australia, Australia

${ }^{8}$ St John, Auckland, New Zealand

${ }^{9}$ Auckland University of Technology, Auckland, New Zealand

${ }^{10}$ Wellington Free Ambulance, Wellington, New Zealand

${ }^{11}$ Queensland Ambulance Service, Brisbane, Queensland, Australia

${ }^{12}$ School of Clinical Sciences, Queensland University of Technology, Brisbane, Queensland, Australia

${ }^{13}$ Emergency and Trauma Centre, The Alfred, Melbourne, Victoria, Australia

${ }^{14}$ University of Washington-Harborview Center for Prehospital Emergency

Care, University of Washington, Seattle, Washington, USA

Twitter Follow Aus-ROC at @Aus_ROC

Acknowledgements The authors would like to acknowledge the late Professor lan Jacobs whose contribution to the science of resuscitation is immeasurable. It was Professor Jacob's vision to establish the Aus-ROC Epistry and it is through his hard work and dedication that the Epistry is now operational. The Aus-ROC Epistry will act as one of his many legacies. The authors would also like to thank the registry staff in each of the participating ambulance services for their contribution.

Collaborators Please see online supplementary appendix 1 for a list of Aus-ROC Steering Committee members.

Contributors BB, JB, KS, TW, HG, CH, MT, AS, TS, BD, AS, MB, EB, KP, PC, GN and JF were all involved in the study design. BB drafted the manuscript and all other authors reviewed and approved the manuscript.

Funding The Australian Resuscitation Outcomes Consortium (Aus-ROC) is a National Health and Medical Research Council (NHRMC) Centre of Research Excellence (\#1029983; https://www.ausroc.org.au/). JB is supported by a cofunded NHMRC/National Heart Foundation (NHF) Fellowship (\#1069985).

\section{Competing interests None declared.}

Ethics approval Monash University Human Research Ethics Committee.

Provenance and peer review Not commissioned; externally peer reviewed.

Open Access This is an Open Access article distributed in accordance with the Creative Commons Attribution Non Commercial (CC BY-NC 4.0) license, which permits others to distribute, remix, adapt, build upon this work noncommercially, and license their derivative works on different terms, provided the original work is properly cited and the use is non-commercial. See: http:// creativecommons.org/licenses/by-nc/4.0/

\section{REFERENCES}

1. Ornato JP, Becker LB, Weisfeldt ML, et al. Cardiac arrest and resuscitation: an opportunity to align research prioritization and public health need. Circulation 2010;122:1876-9.

2. Kouwenhoven WB, Jude JR, Knickerbocker G. Closed-chest cardiac massage. JAMA 1960;173:1064-7.

3. Berdowski J, Berg RA, Tijssen JGP, et al. Global incidences of out-of-hospital cardiac arrest and survival rates: Systematic review of 67 prospective studies. Resuscitation 2010; 81:1479-87.

4. Morrison LJ, Nichol G, Rea TD, et al. Rationale, development and implementation of the Resuscitation Outcomes Consortium Epistry-Cardiac Arrest. Resuscitation 2008;78:161-9.

5. Tijssen JA, Prince DK, Morrison LJ, et al. Time on the scene and interventions are associated with improved survival in pediatric out-of-hospital cardiac arrest. Resuscitation 2015;94:1-7.
6. Daya MR, Schmicker RH, Zive DM, et al. Out-of-hospital cardiac arrest survival improving over time: results from the Resuscitation Outcomes Consortium (ROC). Resuscitation 2015;91:108-15.

7. Zive D, Koprowicz K, Schmidt T, et al. Variation in out-of-hospital cardiac arrest resuscitation and transport practices in the Resuscitation Outcomes Consortium: ROC Epistry-Cardiac Arrest. Resuscitation 2011;82:277-84.

8. The Australian Resuscitation Outcomes Consortium (Aus-ROC). https://www.ausroc.org.au/ (accessed 18 May 2015).

9. Australian Bureau of Statistics. 3235.0-Population by Age and Sex Regions of Australia, 2014. 2014. http://www.abs.gov.au/ AUSSTATS/abs@.nsf/DetailsPage/3235.02014?OpenDocument (accessed 6 Jan 2015)

10. Statistics New Zealand. New Zealand Subnational Population Estimates. 2015. http://nzdotstat.stats.govt.nz/wbos/Index.aspx? DataSetCode=TABLECODE7501\# (accessed 18 Aug 2015).

11. Australian Bureau of Statistics. South Australia (S/T). 2012. http:// stat.abs.gov.au/itt/r.jsp?RegionSummary\&region=4\&dataset=ABS NRP9_ASGS\&geoconcept=REGION\&measure=MEASURE\& datasetASGS=ABS NRP9 ASGS\&datasetLGA=ABS_NRP9 LGA\&regionLGA=REGION\&regionASGS=REGION (accessed 5 May 2015).

12. Australian Bureau of Statistics. Victoria (S/T). 2012. http://stat.abs. gov.au/itt/r.jsp?RegionSummary\&region=2\&dataset=ABS_NRP9_ ASGS\&geoconcept=REGION\&measure=MEASURE\&dataset ASGS $=$ ABS NRP9 ASGS\&datasetLGA=ABS NRP9 LGA\&regionLGA $=$ REGION\&regionASGS=REGION (accessed 5 May 2015).

13. Australian Bureau of Statistics. Western Australia (S/T). 2012. http:// stat.abs.gov.au/itt/r.jsp?RegionSummary\&region=5\&dataset=ABS_ NRP9_ASGS\&geoconcept=REGION\&measure=MEASURE\& datasetASGS=ABS NRP9 ASGS\&datasetLGA=ABS NRP9 LGA\&regionLGA $=$ REGION\&regionASGS=REGION (accessed 5 May 2015).

14. Australian Bureau of Statistics. Queensland (S/T). 2012. http://stat. abs.gov.au/itt/r.jsp?RegionSummary\&region=3\&dataset=ABS REGIONAL_ASGS\&geoconcept=REGION\&measure=MEASURE\& datasetASGS=ABS_REGIONAL_ASGS\&datasetLGA=ABS_ REGIONAL_LGA\&regionLGA=REGION\&regionASGS=REGION (accessed 9 Jan 2015).

15. Statistics New Zealand. New Zealand in Profile: 2014. 2014. http:// www.stats.govt.nz/ /media/Statistics/browse-categories/ snapshots-of-nz/nz-in-profile/2014/nzip-2014-web.pdf (accessed 18 Aug 2015).

16. Greater Wellington Regional Council. Greater Wellington-About the Region. http://www.gw.govt.nz/about-the-region/ (accessed 18 Aug 2015).

17. Australian Bureau of Statistics. 3235.0-Population by Age and Sex, Regions of Australia, 2011. 2011. http://www.abs.gov.au/ausstats/ abs@.nsf/Products/3235.0 2011 Main+Features Western +Australia?OpenDocument (accessed 3 Apr 2015).

18. Australian Bureau of Statistics. 3218.0-Regional Population Growth, Australia, 2013-14. 2013. http://www.abs.gov.au/ausstats/ abs@.nst/mf/3218.0 (accessed 2 Feb 2016).

19. Statistics New Zealand. New Zealand: An Urban/Rural Profile Update. 2006. http://www.stats.govt.nz/browse_for_stats/Maps_and_ geography/Geographic-areas/urban-rural-profile-update.aspx (accessed 2 Feb 2016).

20. Perkins GD, Jacobs IG, Nadkarni VM, et al. Cardiac arrest and cardiopulmonary resuscitation outcome reports: update of the Utstein resuscitation registry templates for out-of-hospital cardiac arrest: a statement for healthcare professionals from a task force of the International Liaison Committee on Resuscitation (American Heart Association, European Resuscitation Council, Australian and New Zealand Council on Resuscitation, Heart and Stroke Foundation of Canada, InterAmerican Heart Foundation, Resuscitation Council of Southern Africa, Resuscitation Council of Asia); and the American Heart Association Emergency Cardiovascular Care Committee and the Council on Cardiopulmonary, Critical Care, Perioperative and Resuscitation. Resuscitation 2015;96:328-40.

21. Nehme Z, Bernard S, Cameron P, et al. Using a cardiac arrest registry to measure the quality of emergency medical service care: decade of findings from the Victorian Ambulance Cardiac Arrest Registry. Circ Cardiovasc Qual Outcomes 2015;8:56-66.

22. Jennings PA, Cameron P, Walker T, et al. Out-of-hospital cardiac arrest in Victoria: rural and urban outcomes. Med J Aust 2006;185:135-9.

23. Nichol G, Thomas E, Callaway CW, et al. Regional variation in out-of-hospital cardiac arrest incidence and outcome. JAMA 2008;300:1423-31. 
24. Ro YS, Shin SD, Song KJ, et al. A trend in epidemiology and outcomes of out-of-hospital cardiac arrest by urbanization level: a nationwide observational study from 2006 to 2010 in South Korea Resuscitation 2013;84:547-57.

25. Fosbøl EL, Dupre ME, Strauss B, et al. Association of neighborhood characteristics with incidence of out-of-hospital cardiac arrest and rates of bystander-initiated CPR: implications for community-based education intervention. Resuscitation 2014;85 1512-17.

26. Hasegawa K, Tsugawa $\mathrm{Y}$, Camargo CA Jr, et al Regional variability in survival outcomes of out-of-hospital cardiac arrest: the All-Japan Utstein Registry. Resuscitation 2013;84:1099-107.

27. Ong MEH, Shin SD, De Souza NNA, et al. Outcomes for out-of-hospital cardiac arrests across 7 countries in Asia: the Pan Asian Resuscitation Outcomes Study (PAROS). Resuscitation 2015;96:100-8.

28. Sasson C, Keirns CC, Smith D, et al. Small area variations in out-of-hospital cardiac arrest: does the neighborhood matter? Ann Intern Med 2010;153:19-22.
29. Callaway CW, Schmicker R, Kampmeyer M, et al. Receiving hospital characteristics associated with survival after out-of-hospital cardiac arrest. Resuscitation 2010;81:524-9.

30. Deasy C, Bray JE, Smith K, et al. Cardiac arrest outcomes before and after the 2005 resuscitation guidelines implementation: evidence of improvement? Resuscitation 2011;82:984-8.

31. Nehme Z, Andrew E, Bernard S, et al. Comparison of out-of-hospital cardiac arrest occurring before and after paramedic arrival: epidemiology, survival to hospital discharge and 12-month functional recovery. Resuscitation 2015;89:50-7.

32. Deasy C, Bernard S, Cameron P, et al. Design of the RINSE Trial: the Rapid Infusion of cold Normal Saline by paramedics during CPR. BMC Emerg Med 2011:11:17.

33. Whitehead L, Perkins GD, Clarey A, et al. A systematic review of the outcomes reported in cardiac arrest clinical trials: the need for a core outcome set. Resuscitation 2015;88:150-7.

34. Gräsner JT, Herlitz J, Koster RW, et al. Quality management in resuscitation-towards a European Cardiac Arrest Registry (EuReCa). Resuscitation 2011;82:989-94. 